\title{
Splitting of Inviscid Fluxes for Real Gases
}

\author{
MeNG-Sing Liou \\ NASA Lewis Research Center, Cleveland. Ohio 44135
}

BRAM VAN LEER

The Lniversity of Michigan, Ann Arbor, Michigan 48109 and Institute for Computational Mechanics in Propulston (ICOMP). NASA Lewis Research Center. Cleveland, Ohio 44135

AND

\section{JIAN-SHUN SHUEN}

Sterdrup Technology, Inc.. Brook Park, Ohio 44135 and NASA Lewis Research Center, Cleveland, Ohio 44135

Received April 1, 1988: revised December 6, 1988

\begin{abstract}
Flux-vector and flux-difference splittings for the inviscid terms of the compressible flow equations are derived under the assumption of a general equation of state for a real gas in equilibrium. No unnecessary assumptions, approximations, or auxihary quantities are introduced. The formulas derived include several particular cases known for ideal gases and readily apply to curvilinear coordinates. Applications of the formulas in a TVD algorithm to onedimensional shock-tube and nozzle problems show their quality znd robustness. if 19y Academic Press. Inc.
\end{abstract}

\section{INTRODUCTION}

Several split-flux formulas are known for upwind differencing of the inviscid terms in CFD codes for ideal gases. The generic formula is due to Gudonov [1] and is based on the exact solution of Riemann's initial-value problem, representing the interaction of two fluid parcels by finite-amplitude waves.

Numerical efficiency justifies the introduction of approximations to the Riemann solution, which leads to various simplifications of the flux formula, accompanied by considerable savings in computational expenses. The most popular "approximate Riemann solvers" are the flux-vector splittings by Steger and Warming [2] and by Van Leer [3] and the flux-difference splittings by Roe [4] and by Osher [5] : see e.g., the review by Harten, Lax and Van Leer [6]. 
With the current interest in high-temperature flows, real-gas effects ${ }^{1}$ must be included, requiring appropriate modifications of all of the above split-flux formulas. Colella and Glaz [7] extended the numerical procedure for obtaining the exact Riemann solution to the real-gas case. (For a review of the conditions under which unique exact solutions to the Riemann problem exist, see Smith [20].) Grossman and Walters [8], as well as Vinokur and Liu [9], extended the formulas of Steger and Warming, Van Leer, and Roe, while Glaister [10] presented an extension of the Roe splitting.

As regards the approximate Riemann solvers for real gases, the derivations in [8] are the least general, introducing unnecessary assumptions, approximations, and auxiliary quantities. A more careful and comprehensive analysis is presented in [9]. Yet, the combined formulas in [8-10] far from exhaust the possibilities; this forms the chief motivation for the present paper.

The present derivation of split-flux formulas has the following features: (1) it includes several particular formulas derived elsewhere for ideal gases, (2) it avoids unnecessary assumptions or approximations, (3) it avoids unnecessary auxiliary quantities, and (4) it readily extends to curvilinear coordinates.

In the next section we briefly discuss the cquation of state (EOS) for the real gas and some related thermodynamics quantities. The detailed derivation of split-flux formulas is given in Section 3. Application of these formulas in a TVD algorithm [11] to one-dimensional shock tube and nozzle problems are presented in Section 4.

\section{Equation of State}

We begin by assuming that the real gas is described by the general equation of state

$$
p=p\left(\rho, e, Y_{i}\right) . \quad i=1,2,3, \ldots
$$

where $p, \rho, e, Y_{i}$ are, respectively, the pressure, density, and specific internal energy of the gas, and the mass fraction of the $i$ th species in the gas. In this paper we shall restrict ourselves to the gas in chemical equilibrium, i.e.,

$$
p=p(\rho, e)
$$

so that derivatives with respect to $Y_{i}$ disappear, simplifying the algebra. Yet, the concepts and algebraic steps needed to describe a non-equilibrium gas are very nearly the same as for the equilibrium gas. In fact, some of the special care in formulating the EOS of an equilibrium gas can be relaxed when including nonequilibrium (finite-rate reaction) effects, because the overall numerical procedure

\footnotetext{
'In this paper the definition of "real gas" is broader than that conventionally used in thermodynamics, by referring to any gas that is not both thermally and calorically perfect.
} 
becomes more straightforward. The computational effort, of course, is increased enormously.

For an ideal gas, $(2.2)$ reduces to $p=(\gamma-1) \rho e$, where $\gamma$ is the ratio of enthaipy $h$ to internal energy $e$, and is a contant.

The speed of sound is

$$
a^{2}=p_{\rho}+p p_{e} / \rho^{2}
$$

Here and in this paper, $p_{p}$ and $p_{e}$ denote the partial derivatives of $p$ with respect to $\rho$ and $e$ while holding other variables fixed.

Several authors [7-9] have advocated the use of an equivalen $\gamma$ for real gases. As in the ideal gas. we define

$$
\eta=h i e, \quad h=\text { specific enthalpy; }
$$

combining this with the definition $h=e+p$ ives

$$
p=(\gamma-1) \rho e \text {. }
$$

This is identical in form to the EOS of the ideal gas, but now we have

$$
\gamma=\gamma(\rho, e) \text {. }
$$

We note that, although the range of variation of $\gamma$ may be limited, this variation may be of great significance. Since it is the factor $(y-1)$ that appears in the equations, a small change in $\gamma$ can result in an appreciable change in $p$; e.g., going fron $\gamma=1.1$ to $\gamma=1.2$ doubles the pressure. On the other hand, changes in flow variables of several orders of magnitude can occur with $\gamma$ fixed, as in the case of an ideal gas. Note that for a real gas $\gamma$ in general will not be a monotone function of temperature.

The speed of sound expressed in terms of $\because$ now has the form

$$
a^{2}=\eta p / \rho+e\left[(\eta-1) e_{\ell}+\rho \gamma_{\rho}\right]
$$

where $\gamma_{c}=\lambda \gamma_{i} \hat{c}$ and $\gamma_{\rho}=\partial \gamma / \hat{c} \rho$. For an ideal gas $(2.6 a)$ reduces to $a^{2}=\gamma p p^{\prime}$, which motivates the introduction of an auxiliary variable $\Gamma$ such that

$$
a^{2}=\Gamma p / \rho=\Gamma(\eta-1) \rho
$$

where $\Gamma=\Gamma(\rho, e)$.

We caution that the appearance of these different "equivalent $\gamma$ "s" may at times add more confusion than insight.

From thermodynamic principles, it is possible to caiculate any thermodynamic variable for each pair of state quantities $(\rho, e)$. In practice, a table or a least-square fitted surface is generated a priori for reasons of computational efficiency. We adopt the latter approach because interpolation is avoided, resulting in greater efficiency. However, care must be taken to ensure, while fitting, not only that the error is kept within reasonable bounds but also that no numerical oscillations are introduced. 


\section{Construction of Split FluXes}

For the construction of split-flux formulas, we first need to consider the 1D Euler equations,

$$
\frac{\partial \mathbf{U}}{\partial t}+\frac{\partial \mathbf{F}(\mathbf{U})}{\partial x}=0
$$

where $\mathbf{U}^{t}=[\rho, \rho u, \rho E]$ and $\mathbf{F}^{t}=\left[\rho u, \rho u^{2}+p .(\rho E+p) u\right]$.

As indicated previously, the EOS now is expressed in terms of all variables $\mathbf{U}$, viz. as $p=p(\rho(\mathbf{U}), e(\mathbf{U}))$. The Jacobian matrix of the flux can be separated into two parts, i.e.,

$$
\mathbf{A}=\frac{\partial \mathbf{F}}{\partial \mathbf{U}}=\mathbf{A}_{e}+\mathbf{A}_{\rho}
$$

where

$$
\mathbf{A}_{e}=\left(\begin{array}{ccc}
0 & 1 & 0 \\
-u^{2}\left(2-p_{e} / \rho\right) / 2+\left(p-p_{e} e\right) / \rho & u\left(2-p_{e} / \rho\right) & p_{e} / \rho \\
u\left[-H+u^{2} p_{e} / 2 \rho+\left(p-p_{e} e\right) / \rho\right] & H-u^{2} p_{e} / \rho & u\left(1+p_{e} / \rho\right)
\end{array}\right)
$$

contains the derivative $p_{e}$, and the matrix containing $p_{\rho}$ is

$$
\mathbf{A}_{\rho}=\left(\begin{array}{ccc}
0 & 0 & 0 \\
p_{\rho}-p / \rho & 0 & 0 \\
u\left(p_{\rho}-p / \rho\right) & 0 & 0
\end{array}\right)
$$

the quantity $H=E+p / \rho$ is the specific total enthalpy.

The eigenvalues of these matrices are respectively

$$
\begin{aligned}
& \lambda(\mathbf{A})=u-a, \quad u, \quad u+a, \\
& \lambda\left(\mathbf{A}_{e}\right)=u-a_{e}, \quad u, \quad u+a_{e}, \\
& \lambda\left(\mathbf{A}_{\rho}\right)=0, \quad 0, \quad 0,
\end{aligned}
$$

where $a_{e}{ }^{2}=p\left(p_{e} / \rho+1\right) / \rho=a^{2}-\left(p_{\rho}-p / \rho\right)$. Thus, the matrices $\mathbf{A}$ and $\mathbf{A}_{e}$ have a complete set of eigenvectors, but $\mathbf{A}_{\rho}$ does not. We note that $a_{e}=a$ and $\mathbf{A}_{e}=\mathbf{A}$ for a gas in which $p$ depends linearly on $\rho$, viz. a thermally perfect gas. Since the matrix A has a complete set of eigenvectors, it can be readily diagonalized by a similarity matrix $\mathbf{S}$ whose column vectors are the right eigenvectors of $\mathbf{A}$ :

$$
\mathbf{A}=\mathbf{S} \Lambda \mathbf{S}^{-1}, \quad \operatorname{diag} \Lambda=\lambda(\mathbf{A}) .
$$

Furthermore, we find that the flux can be written as

$$
\mathbf{F}=\mathbf{F}_{h}+\mathbf{F}^{\prime}
$$


where

$$
\mathbf{F}_{h}=\mathbf{A} \mathbf{U}
$$

and

$$
\mathbf{F}^{\prime}=\mathbf{A}_{\rho} \mathbf{U}
$$

That is, the flux vector $\mathbf{F}$ no longer possesses the property of homogeneily but, rather, is the sum of a homogeneous and an inhomogeneous part.

\subsection{Steger-Warming Splitting}

By splitting according to the sign of the eigenvalues, i.e.,

$$
\Lambda=\Lambda^{+}+\Lambda^{-}
$$

and, correspondingly,

$$
\mathbf{A}=\mathbf{A}^{+}+\mathbf{A}^{-}
$$

a real-gas version of Steger-Warming flux-vector splitting for $F_{h}$ is obtained, wh

$$
\mathbf{F}_{h}=\mathbf{F}_{h}^{+}+\mathbf{F}_{h}^{-}=\mathbf{A}^{+} \mathbf{U}+\mathbf{A}^{-} \mathbf{U}
$$

These split fluxes are suited for upwind differencing. Note that. unlike in [8]. the tue speed of sound $a$ of the gas is used to determine the switching in $(3.6)$. Since $A_{p}$ does not have a complete set of eigenvectors, an equation that includes $F^{\prime}$ alone does not have a hyperbolic character. In consequence, central differencing, leading to the final splitting

$$
\mathbf{F}^{ \pm}=\mathbf{F}_{h}^{ \pm}+\frac{1}{2} \mathbf{F}^{\prime}
$$

may be appropriate. This has been verified in the numerical experiments of Section 4.

An entirely different approach, bascd on the "bcam scheme" [12], is described in [9]. A discussion and numerical comparison of the various real-gas versions of the Steger-Warming splitting is of limited interest, since this formula, among all splitfux formulas, has been shown in various studies (see, e.g., [13]) to yield the leasi accurate solutions in the ideal-gas case. The formula derived above is as efficient as any real-gas version can be and appears satisfactory in practice.

One cause of inaccuracy in the Steger-Warming splitting is that the split-1in derivatives are discontinuous when any of the eigenvalues $\lambda(\mathbf{A})$ changes sign. This gives rise to "glitches" in numerical solutions, unless some extra smoothing is built into the flux formula. To solve this problem radically, Van Leer [3] developed continuously differentiable splitting. 


\subsection{Van Leer Splitting}

In this section we present a comprehensive derivation of the Van Leer splitting that includes a family of flux choices and is easily applicable to curvilinear coordinates. It further is independent of the EOS used and does not require homogeneity of the flux vector. Splitting takes place only when there exist eigenvalues of mixed signs, i.e., when $M^{2}<1$ for the system (3.1) with eigenvalues (3.3a); $M$ is the local Mach number. Since $u=M a$, the mass flux $\mathbf{F}_{1}=\rho a M$ is split as

$$
\begin{aligned}
F_{1} & =F_{1}^{+}+F_{1}^{-}, \\
F_{1}^{ \pm} & = \pm \frac{1}{4} \rho a(M \pm 1)^{2} .
\end{aligned}
$$

Note that the split fluxes $F_{\overline{1}}^{\perp}$ have vanishing slope as $M \rightarrow \mp 1$, yielding smooth switching. This forms the basis for splitting the remaining fluxes, namely, for expressing $F_{2}$ and $F_{3}$ in terms of $F_{1}^{ \pm}$. Let us write

$$
F_{2}=\rho u^{2}+p=(\rho u a) M+p
$$

where the first term on the RHS, representing the convection of momentum, already has the desired factor,

$$
M=\left[(M+1)^{2}-(M-1)^{2}\right] / 4 .
$$

Now we must see if $p$ can be written in terms of $(M+1)^{2}$ and $(M-1)^{2}$. Let us try the combination,

$$
p=x\left[(M+1)^{2}-(M-1)^{2}\right]+y\left[(M+1)^{2}+(M-1)^{2}\right]=4 x M+2 y\left(M^{2}+1\right) .
$$

This leaves us one equation for two unknowns $x$ and $y$. By choosing

$$
2 y=p
$$

which must be true anyway for $M=0$, we find

$$
x=-\frac{1}{4} p M
$$

for arbitrary $M$. Thus $p$ is recast as

$$
p=\frac{p}{\rho a^{2}}\left[F_{1}^{+}(-u+2 a)+F_{1}^{-}(-u-2 a)\right] .
$$

Substitution in (3.8a) yields the splitting of $F_{2}$,

$$
\begin{aligned}
& F_{2}=F_{2}^{+}+F_{2}^{-}, \\
& F_{2}^{ \pm}=F_{1}^{ \pm}\left[u-\frac{p}{\rho a^{2}}(u \mp 2 a)\right]=F_{1}^{ \pm}[u-(u \mp 2 a) / \Gamma] .
\end{aligned}
$$


We note that the last equality is obtained by the use of definition (2.6b) and is identical to the formula for an ideal gas.

We turn now to the splitting of the energy flux $F_{3}$. The fiux, again, contains a convection term,

$$
F_{3}=\rho u E+p u=(\rho a E) M+p u
$$

where the factor $M$ can be represented as in (3.8b). Since $p$ has factors $(u \pm 2 a)$, it is natural to assume a quadratic function in $u$ for $p u$; for symmetry reasons this must have the form

$$
\begin{aligned}
p u & =F_{1}^{+}\left(l u^{2}+2 m u a+n a^{2}\right)+F_{1}^{-}\left(l u^{2}-2 m u a+n a^{2}\right) \\
& =(l+m) \rho u^{3}+(m+n) \rho u a^{2} .
\end{aligned}
$$

This leaves us many possibilities. We choose to eliminate $\rho u^{3}$ by letting

$$
l+m=0
$$

hence

$$
m+n=p i \rho a^{2}
$$

Consequently, a lamily of $\infty$ choices, with single parameter $m$, results for $F_{3}$.

$$
\begin{aligned}
& F_{3}=F_{3}^{+}+F_{3}^{-}, \\
& F_{3}^{ \pm}=F_{1}^{ \pm}\left[H-m(u \mp a)^{2}\right] .
\end{aligned}
$$

The identical result is obtained when letting $(m+m)$ vanish, instead of $(l+n ?)$. because of the symmetric occurrence of $u^{2}$ and $a^{2}$. Van Leer's splitting is a member of this family, found by requiring that the terms in the square bracket form a perfect square. This leads to

$$
m=\frac{h / a^{2}}{1+2 h i a^{3}}
$$

and

$$
F_{3}^{ \pm}=\frac{1}{2} F_{1}^{ \pm} \frac{(u a \pm 2 h)^{2}}{\left(a^{2}+2 h\right)} \text {. }
$$

For an ideal gas, this results in the nice property that one eigenvalue of each spliflux Jacobian vanishes, because $F_{3}^{+}$depends solely on $F_{1}^{ \pm}$and $F_{2}^{ \pm}$:

$$
F_{3}^{ \pm}=\operatorname{const} \frac{\left(F_{2}^{ \pm}\right)^{2}}{F_{1}^{ \pm}} \text {. }
$$


As a result, numerical diffusion is minimal and sharp steady shocks can be obtained [14]. This property, however, is lost for the real gas. The particular eigenvalues still are very close to zero and may be either positive or negative. In the former case the formula has slightly increased dissipation; in the latter case it might make upwind differencing unstable. Neither effect is noticeable in practice; see numerical tests in Section 4. A full stability analysis of upwind schemes based on the above flux-vector splittings is complicated by the property that the Jacobians of $\mathbf{F}^{+}$and $\mathbf{F}^{-}$do not commute. It is conceivable that the overall schemes are linearly stable, although the individual eigenvalues suggest otherwise. Another obvious choice is

$$
\begin{aligned}
m & =0, \\
F_{3}^{ \pm} & =F_{1}^{ \pm} H .
\end{aligned}
$$

This has the advantage of being the simplest and most efficient formula possible; the ideal-gas version has one eigenvalue close to zero and with correct sign. It was derived independently by Hänel et al. [15].

In the derivation of Vinokur and Liu [9], the one-parameter family of formulas is governed by the parameter $\psi$ in the split energy flux:

$$
F_{3}^{ \pm}=F_{1}^{ \pm}\left\{\frac{[(\Gamma-1) u \pm 2 a]^{2}}{2\left(\Gamma^{2}-1\right)}+\left(h-\frac{a^{2}}{\Gamma-1}\right)+\psi(u \mp a)^{2}\right\} .
$$

The first term corresponds to Van Leer's flux for an ideal gas; the second term vanishes for an ideal gas. Vinokur and Liu [9] therefore choose $\psi=0$ as the extension of Van Leer's scheme to the real-gas case. Our extension (3.13b) corresponds to

$$
\psi=\frac{1-(\Gamma-1)\left(h / a^{2}\right)}{(\Gamma+1)\left(1+2 h / a^{2}\right)}
$$

while (3.14) is generated by

$$
\psi=\frac{1}{\Gamma+1}
$$

Clearly, the two formulas (3.12) and (3.15a) generate the same split energy fluxes. Our formula, however, does not favor the original Van Ieer flux and therefore may be more convenient in searching for other special cases.

When extending the split fluxes (3.10) and (3.12) to curvilinear multidimensional moving coordinates, we benefit from the fact that $p$ and $p u$ have been expressed in terms of $F_{1}^{ \pm}$. The derivation is straightforward and yields the following formulas for the split fluxes in the direction of a coordinate $\xi$ : 


$$
\begin{aligned}
& F_{1}^{ \pm}= \pm \frac{1}{4} \frac{\rho}{a}(\tilde{u} \pm a)^{2}= \pm \frac{1}{4} \rho a(\tilde{M} \pm 1)^{2}, \\
& F_{2}^{ \pm}=F_{1}^{ \pm}\left[u-\xi_{x}(\tilde{u} \mp 2 a) / \Gamma\right], \\
& F_{3}^{ \pm}=F_{1}^{ \pm}\left[v-\xi_{y}(\tilde{u} \mp 2 a) / l\right], \\
& F_{4}^{ \pm}=F_{1}^{ \pm}\left[w-\zeta_{-}(\tilde{u} \mp 2 a) / \Gamma\right], \\
& F_{5}^{ \pm}=F_{1}^{ \pm}\left[H+\xi_{r}(\tilde{u} \mp 2 a) / \Gamma-m(\tilde{u} \mp 2 a)^{2}\right],
\end{aligned}
$$

Here $u, v$, and $w$ are the Cartesian velocity components and $\tilde{u}$ is the contravariant $\xi$-velocity, i.e., the velocity normal to a moving surface of constant $\xi ; \tilde{M}$ is the corresponding contravariant Mach number. The form is the same as for the ideal gas except that $\Gamma$ instead of $\gamma$ is used; see Anderson, Thomas, and Rumsey [16], and also Parpia [21].

Note that none of the differentiable splittings derived above involves special derivatives of the EOS. The only thermodynamic quantities needed are $p$ and $a$. which are standard. Unlike in [8], no assumption of homogeneity of the flux ${ }^{2}$ is required.

\subsection{Roe Spliting}

To construct Roe's flux-difference splitting, one usually defines an average state U such that

$$
\begin{aligned}
\Delta \mathbf{F} & =\hat{\mathbf{A}} \Delta \mathbf{U}, \\
\hat{\mathbf{A}} & =\mathbf{A}(\hat{\mathbf{U}}), \\
\hat{\mathbf{U}} & =\hat{\mathbf{U}}\left(\mathbf{U}_{L}, \mathbf{U}_{R}\right),
\end{aligned}
$$

where

$$
\Delta(\cdot)=(\cdot)_{R}-(\cdot)_{L}
$$

and the matrix $\mathbf{A}$,

$$
\begin{aligned}
\mathbf{A}(\mathbf{U}) & =\frac{\partial \mathbf{F}}{\partial \mathbf{U}} \\
& =\left(\begin{array}{ccc}
0 & 1 & 0 \\
-u^{2}\left(2-p_{e} / \rho\right) / 2+\left(p_{\rho}-p_{e} e / \rho\right) & u\left(2-p_{e} / \rho\right) & p_{e} i \rho \\
u\left[-H+u^{2} p_{e} / 2 \rho+\left(p_{\rho}-p_{e} e / \rho\right)\right] & H-u^{2} p_{e}^{i} \rho & \left.u\left(i+p_{e} / \rho\right)\right)
\end{array}\right)
\end{aligned}
$$

has precisely the same form as in $(3.2 \mathrm{a})-(3.2 \mathrm{c})$. Note that the difference between states " $L$ " and " $R$ " is not necessarily small.

The object now is to find an average state such that $(3.17 \mathrm{a})$ is satisfied exactly 
for all admissible pairs $\left(\mathbf{U}_{L}, \mathbf{U}_{R}\right)$. In the ideal-gas case this is easily accomplished since (3.17a) represents three relatively simple equations with three unknowns, the components of $\hat{\mathbf{U}}$. (Note, for instance, that the term $\left(p_{\rho}-p_{e} e / \rho\right)$ appearing in the first column of $\mathbf{A}$ vanishes.) In fact, the matrix $\mathbf{A}(\hat{\mathbf{U}})$ is completely determined by only two average state quantities, as the density does not occur explicitly. The average Jacobian depends only on the quantities $\hat{u}$ and $\hat{H}$; an average density $\hat{\rho}$ may be chosen freely, although there is an obvious choice for it (śee (3.20c)).

For a real gas, the simplicity is lost. The nonlinear system (3.17a) must be solved numerically and in general may have more than one solution or no solution at all. In order to find a practical formula for $\hat{\mathbf{A}}$ we must relax the constraint (3.17b) and allow independent averages of more than three state quantities to enter the elements of $\hat{\mathbf{A}}$. Judging from the form of $\mathbf{A}$ in (3.18) we have six non-constant elements to play with, so there is room for six independent averages. We shall choose the set $\left(\hat{u}, \hat{\rho}, \hat{e}, \hat{H}, \hat{p}_{e}, \hat{p}_{\rho}\right)$. We note that the relation of the total enthalpy to the other flow quantities, i.e.,

$$
H=e+p / \rho+u^{2} / 2
$$

while holding pointwise, does not necessarily hold for the average state quantities, which are functions of two states. Similarly, while $p=p(\rho, e), \hat{p}$ is not obtained by substituting $(\hat{\rho}, \hat{e})$ in the $\operatorname{EOS}$, but rather must be defined separately; this is also the case for $\hat{p}_{\rho}$ and $\hat{p}_{e}$. In the following derivation there is no need for an explicit definition of $\hat{p}$. We note that the analysis from (3.19a) to (3.24b) was also independently derived by Glaister [10].

The mass-flux equation in (3.17a) is automatically satisfied for any average state. To satisfy the momentum-flux equation, we choose

$$
\begin{aligned}
& \text { (i) } \Delta \rho u=\hat{\rho} \Delta u+\hat{u} \Delta \rho \text {; } \\
& \text { (ii) } \Delta \rho u^{2}=2 \hat{\rho} \hat{u} \Delta u+\hat{u}^{2} \Delta \rho .
\end{aligned}
$$

Let the Roe-average operator $\mu$ be defined as

$$
\mu(f)=\frac{f_{L}+r f_{R}}{1+r}, \quad r=\left(\rho_{R} / \rho_{L}\right)^{1,2} .
$$

The conditions (i) and (ii) are met by

$$
\begin{aligned}
& \hat{u}=\mu(u), \\
& \hat{\rho}=r \rho_{L} .
\end{aligned}
$$

Assuming further that

$$
\text { (iii) } \Delta \rho e=\hat{\rho} \Delta e+\hat{e} \Delta \rho
$$


which is met by

$$
\hat{e}=\mu(e)
$$

the second equation of $(3.17 \mathrm{a})$ becomes

$$
\begin{aligned}
& \operatorname{RHS}_{2}=\Delta \rho u^{2}+\left(\hat{p}_{e} \Delta e+\hat{p}_{p^{2}} \Delta \rho\right) \\
& \mathrm{LHS}_{2}=\Delta \rho u^{2}+\Delta p .
\end{aligned}
$$

Clearly, the condition to be satisfied is

$$
\text { (iv) } \Delta p=\hat{p}_{e} \Delta e+\hat{p}_{p} \Delta \rho \text {. }
$$

Finally, we find that the energy-flux equation is readily satisfied by setting

$$
\text { (v) } \quad \Delta \rho u H=\hat{\rho} \hat{H} \Delta u+\hat{u} \Delta \rho \hat{H} \text {; }
$$

hence

$$
\hat{H}=\mu(H)
$$

Left to be completed are the definitions of $\hat{p}_{\mathrm{c}}$ and $\hat{p}_{p}$. Glaister $[10]$ suggests

$$
\begin{aligned}
& \hat{p}_{e}=\frac{p_{E}-p_{w}}{\Delta e}, \\
& \hat{p}_{0}=\frac{p_{S}-p_{N}}{\Delta \rho} .
\end{aligned}
$$

where $p_{E}, p_{W}, p_{S}$, and $p_{N}$ are approximated at points snown in ihe sketch by algebraic averaging

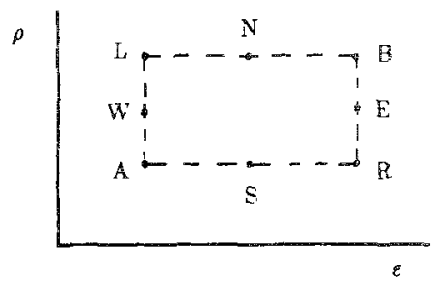

i.e.

$$
\begin{array}{ll}
p_{E}=\frac{1}{2}\left(p_{R}+p_{B}\right), & p_{B}=p\left(p_{L}, e_{R}\right), \\
p_{H}=\frac{1}{2}\left(p_{L}+p_{A}\right), & p_{A}=p\left(\rho_{R}, e_{L}\right), \\
p_{N}=\frac{1}{2}\left(p_{L}+p_{B}\right), & \\
p_{S}=\frac{1}{2}\left(p_{R}+p_{A}\right) . &
\end{array}
$$


With this choice, (3.22) is met precisely. However, the artificially introduced states, $A$ and $B$, cause problems for discontinuities, in particular for a contact discontinuity across which the density and internal energy jump appreciably. If the EOS is a non-convex function (as for an ideal gas) and $L$ and $R$ are two admissible discrete states, then $A$ and $B$ can lie outside the region of validity of the EOS. This will manifest itself in a calculation in the form of oscillations near the discontinuity, and/or an inaccurate solution.

Below we propose a family of formulas that use derivative information from the average state $(\hat{\rho}, \hat{e})$, thus yielding greater consistency among the independently averaged quantities. Specifically, we introduce

$$
\bar{p}_{\rho}=p_{\rho}(\hat{\rho}, \hat{e}), \quad \bar{p}_{e}=p_{e}(\hat{\rho}, \hat{e}) .
$$

Next, we need a density unit $R$ and an energy unit $E$ to make all quantities involved non-dimensional. Equation (3.22) may then be written as

$$
\left(\frac{\Delta e}{\Delta p} R\right) \frac{\hat{p}_{e}}{R}+\left(\frac{\Delta \rho}{\Delta p} E\right) \frac{\hat{p}_{\rho}}{E}=1
$$

or, simply,

$$
\alpha x+\beta y=1
$$

with

$$
\begin{array}{ll}
x \equiv \frac{\Delta l}{\Delta p} R, & \beta \equiv \frac{\Delta \rho}{\Delta p} E, \\
x \equiv \frac{\hat{p}_{\epsilon}}{R}, & y \equiv \frac{\hat{p}_{\rho}}{E} .
\end{array}
$$

The set of values $\left(x_{0}, y_{0}\right) \equiv\left(\bar{p}_{e} / R, \bar{p}_{\rho} / E\right)$ in general does not satisfy $(3.25 \mathrm{~b})$; i.e., the point $Q \equiv\left(x_{0}, y_{0}\right)$ in the $(x, y)$-plane does not lie on the line $l$ represented by $(3.25 \mathrm{~b})$; see the sketch.

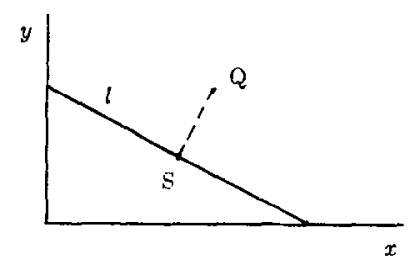

The point on $l$ closest to $Q$ is the projection $S$ of $Q$ onto $l$; this is the optimal choice regarding consistency of $\left(\hat{p}_{e}, \hat{p}_{\rho}\right)$ with $\left(\bar{p}_{e}, \bar{p}_{\rho}\right)$, for the given distance scaling $(R, E)$. The coordinates of $S$ are formed by combining $(3.25 \mathrm{~b})$ with the equation for $S Q$, viz.

$$
\beta\left(x-x_{0}\right)-\alpha\left(y-y_{0}\right)=0
$$


yielding

$$
\begin{aligned}
& x_{S}=\frac{\beta^{2} x_{0}-\alpha \beta y_{0}+\alpha}{\alpha^{2}+\beta^{2}}, \\
& y_{S}=\frac{\alpha^{2} y_{0}-\alpha \beta x_{0}+\beta}{\alpha^{2}+\beta^{2}} .
\end{aligned}
$$

An obvious scaling is

$$
R=\frac{\Delta p}{\Delta e}, \quad E=\frac{\Delta p}{\Delta \rho}
$$

yielding $\alpha=1, \beta=1$; this leads to the values

$$
\begin{aligned}
& \hat{p}_{e}=\frac{1}{2}\left[\left(A p-\bar{p}_{\rho} A \rho\right) / A e+\bar{p}_{e}\right] \\
& \hat{p}_{\rho}=\frac{1}{2}\left[\left(\Delta p-\bar{p}_{e} \Delta e\right) / \Delta \rho+\bar{p}_{\rho}\right]
\end{aligned}
$$

All of the numerical results presented in Section 4 for flux-difference splitting were obtained with this choice. It does have a flaw. though: when $A e$ or $1 \rho$ vanishes, $\hat{p}_{g}$ or $\dot{p}_{\rho}$ becomes indeterminate (when both $A e$ and $A \rho$ vanish there is no jump and. therefore, no problem). Note that if $\Delta e$ or $\Delta \rho$ vanishes, $\vec{p}_{e}$ simply reduces to $\bar{p}_{e}$ or $\hat{p}_{p}$ to $\bar{p}_{p}$.

A better scaling is

$$
R=\bar{p}_{e}, \quad E=\bar{p}_{\rho}
$$

we then have $\left(x_{0}, y_{0}\right)=(1,1)$, yielding

$$
\begin{aligned}
& x_{S}=1-\alpha \frac{\alpha+\beta-1}{\alpha^{2}+\beta^{2}}, \\
& y_{S}=1-\beta \frac{\alpha+\beta-1}{\alpha^{2}+\beta^{2}}
\end{aligned}
$$

or

$$
\begin{aligned}
& \hat{p}_{e}=\bar{p}_{e}\left\{1-\frac{\bar{p}_{e} \Delta e}{\left(\bar{p}_{e} \Delta e\right)^{2}+\left(\bar{p}_{\rho} \Delta \rho\right)^{2}}\left(\bar{p}_{e} \Delta e+\bar{p}_{\nu} \Delta \rho-\Delta p\right)\right\}, \\
& \hat{p}_{p}=\bar{p}_{p}\left\{1-\frac{\bar{p}_{p} \Delta \rho}{\left(\bar{p}_{e} \Delta e\right)^{2}+\left(\bar{p}_{\rho} \Delta \rho\right)^{2}}\left(\bar{p}_{e} \Delta e+\bar{p}_{p} \Delta \rho-\Delta p\right)\right\} .
\end{aligned}
$$

Note further that

$$
\hat{\partial p}=A p-\bar{p}_{e} A e-\bar{p}_{\rho} A \rho
$$


is the residual of $(3.22)$ when we substitute $\left(\bar{p}_{e}, \bar{p}_{\rho}\right)$ for $\left(\hat{p}_{e}, \hat{p}_{\rho}\right)$; we may rewrite $(3.28 \mathrm{c})$ as

$$
\begin{aligned}
& \hat{p}_{e} \Delta e=\bar{p}_{e} \Delta e+\omega \delta p \\
& \hat{p}_{\rho} \Delta \rho=\bar{p}_{\rho} \Delta \rho+(1-\omega) \delta p
\end{aligned}
$$

with

$$
\omega=\frac{\left(\bar{p}_{e} \Delta e\right)^{2}}{\left(\bar{p}_{e} A \rho\right)^{2}+\left(\bar{p}_{\rho} A \rho\right)^{2}} .
$$

In words: the residual $\delta p$ is distributed over the terms $\hat{p}_{e} \Delta e$ and $\hat{p}_{\rho} \Delta \rho$ in (3.22) with weights $\omega$ and $1-\omega$. In the case that $\Delta e$ or $\Delta \rho$ vanishes, $\omega$ or $1-\omega$ vanishes. It is evident from (3.29) that choosing a different scaling $(R, E)$ just amounts to choosing different weights $\omega$ and $1-\omega$ for distributing the residual of (3.22) over its constituent terms. The choice (3.27), for example, amounts to simply taking $\omega=1-\omega=\frac{1}{2}$. Another choice that comes to mind is

$$
\omega=\frac{\left|\bar{p}_{e} \Delta e\right|}{\left|\bar{p}_{\epsilon^{\prime}} \Delta e\right|+\left|\bar{p}_{\rho} \Delta \rho\right|}
$$

which, like $(3.29 b)$, has the desirable property that $\omega$ vanishes with $\Delta e$, and $1-\omega$ with $\Delta \rho$.

All formulas presented above return the standard values $\left(\hat{p}_{e}, \hat{p}_{\rho}\right)=\left(\bar{p}_{e}, \bar{p}_{\rho}\right)$ for a calorically perfect gas, since in this case $Q$ lics on $l$. The above analysis uses the distance from $\left(\hat{p}_{e}, \hat{p}_{\rho}\right)$ to $\left(\bar{p}_{e}, \bar{p}_{\rho}\right)$ as the relevant measure; obviously, other strategies are possible. The formulas suggested by Vinokur and Liu [9] are based on a different choice of independently averaged quantities, with different constraints, and are more complicated.

The Roe splitting itself is based, once again, on (3.6b).

$$
\Delta \mathbf{F}=(\Delta \mathbf{F})^{+}+(\Delta \mathbf{F})^{-}=\hat{\mathbf{A}}^{+} \Delta \mathbf{U}+\hat{\mathbf{A}}^{-} \Delta \mathbf{U}=\hat{\mathbf{A}} \Delta \mathbf{U}
$$

for its implementation the eigenvalues $\lambda(\hat{\mathbf{A}})$ and the eigenvectors arranged in the similarity matrix $\mathbf{S}(\hat{\mathbf{A}})$ must be known; cf. (3.4). If we derive the average sound speed $\hat{a}$ from the independently averaged quantities, using (2.3) and (3.18), i.e.,

$$
\hat{a}^{2}=\hat{p}_{\rho}+\frac{\hat{p}_{e}}{\hat{\rho}}\left(\hat{H}-\hat{e}-\frac{1}{2} \hat{u}^{2}\right)
$$

the point-wise formulas for $\lambda(\mathbf{A})$ and $\mathbf{S}(\mathbf{A})$ carry over to the case of averaged input quantities. The eigenvalues of $\hat{\mathbf{A}}$ indeed are $\hat{u}, \hat{u} \pm \hat{a}$ (cf. (3.3a)), and the right eigen- 
vectors of $\hat{\mathbf{A}}$ (the column vectors of $\mathbf{S}$ ) follow exactly by inserting the hatted quantities into the point-wise formula:

$$
\mathbf{S}=\left(\begin{array}{ccc}
1 & 1 & 1 \\
u & u+a & u-a \\
H-\frac{\rho}{p_{e}} a^{2} & H+u a & H-u a
\end{array}\right)
$$

\section{Numerical Test}

Extensive tests over a wide range of flow conditions have been conducted to validate the accuracy of the present formulation. Some extreme cases of $1 D$ unsteady shock tube and steady nozzle problems are presented in this paper; the performance of the split-flux schemes are compared with the exact solution. The result of accounting for real-gas effects is discussed.

The Euler equations are integrated using the explicit Lax-Wendroff scheme. To obtain a crisp and monotone shock representation, the TVD scheme based on the above split formulas, as described in [11]. is employed, along with the super-Bee limiter [17] for steepening of the contact discontinuity.
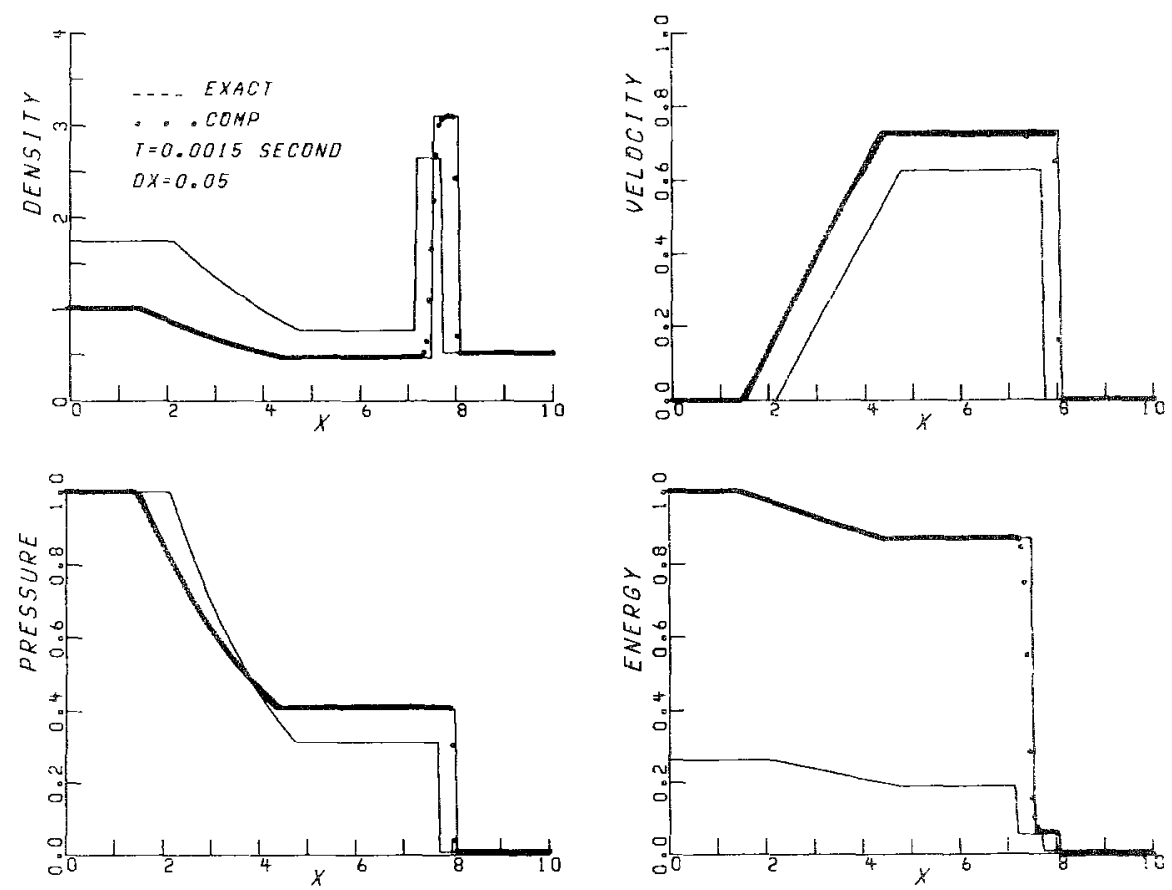

FIG. 1. Shock tube problem, Roe flux-difference splitting. 
The computation domain consists of 200 and 100 equally-spaced intervals for the shock-tube and nozzle problems, respectively.

\subsection{Shock-Tube Problem}

The initial conditions are those used in [8]:

For $0 \leqslant x \leqslant 5$,

$$
\begin{aligned}
& p_{4}=100 \mathrm{~atm}, \\
& T_{4}=9000 \mathrm{~K}, \\
& u_{4}=0 ;
\end{aligned}
$$

And $5 \leqslant x \leqslant 10$,

$$
\begin{aligned}
& p_{1}=1 \mathrm{~atm}, \\
& T_{1}=300 \mathrm{~K}, \\
& u_{1}=0 .
\end{aligned}
$$

The EOS is generated using the widely referred program by Gordon and McBride [18] for equilibrium air in the range $250 \mathrm{~K} \leqslant T \leqslant 12000 \mathrm{~K}$,
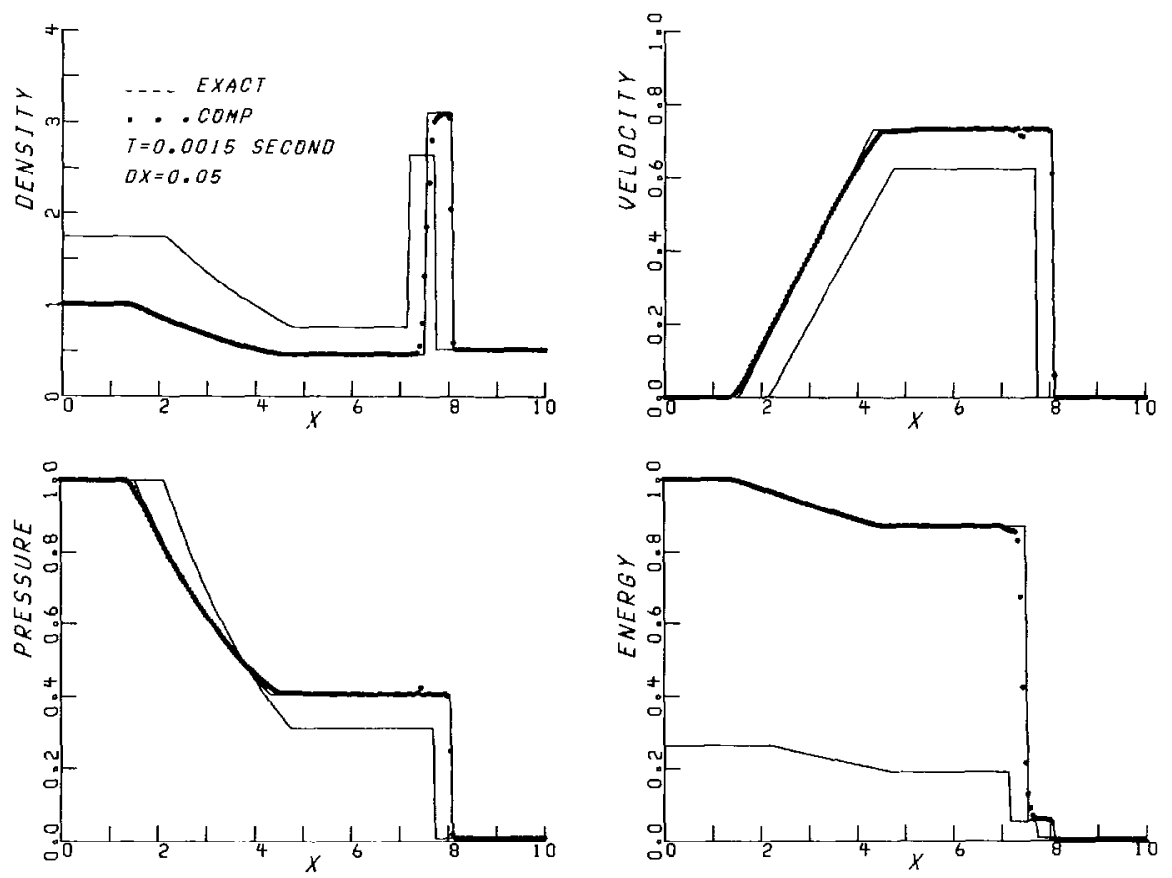

FIG. 2. Shock tube problem, Steger-Warming flux-vector splitting. 
$0.1 \mathrm{~atm} \leqslant p \leqslant 100 \mathrm{~atm}$, in which 17 species are included. With over 3600 sets of state points, a least-squares fit for pressure is obtained with 20 basis functions of $(\rho, e)$. The resulting standard deviation does not exceed $0.2 \%$; the maximum value occurs. as expected, on the boundary.

The procedure for finding the "exact" solution basically is similar to that of $[7]$ except for differences in the details of the numerical steps. We iterate the system via Newton's procedure; the pressure behind the shock $p_{2}$ is iterated until the velocity integrated through the rarefaction fan and the velocity obtained from the jump relation across the shock are equal at the contact discontinuity. The procedure converges in only a few iterations.

Figures 1-3 show the numerical results of the Roe. Steger-Warming, and Van Leer spiittings for, respectively, $\rho / \rho_{4}, u / u_{4}, p / p_{4}$, and $e / e_{4}$, together with exact solutions for real and ideal gases. The jump across the contact is rather large, about one order of magnitude in density and energy. This is a difficult case to calculate, as the initial temperatures differ by a factor 30 and, consequently, the compositions of the air are completely different. It has been our experience that the TVD scheme can handle large differences in pressure very well, but not as well if there also are large temperature differences. Nevertheless, our numerical results generally show excellent agreement with the exact solution. A crisp shock profile is seen, while the effect of dissipation at the contact discontinuity remains as in the case of the ideal
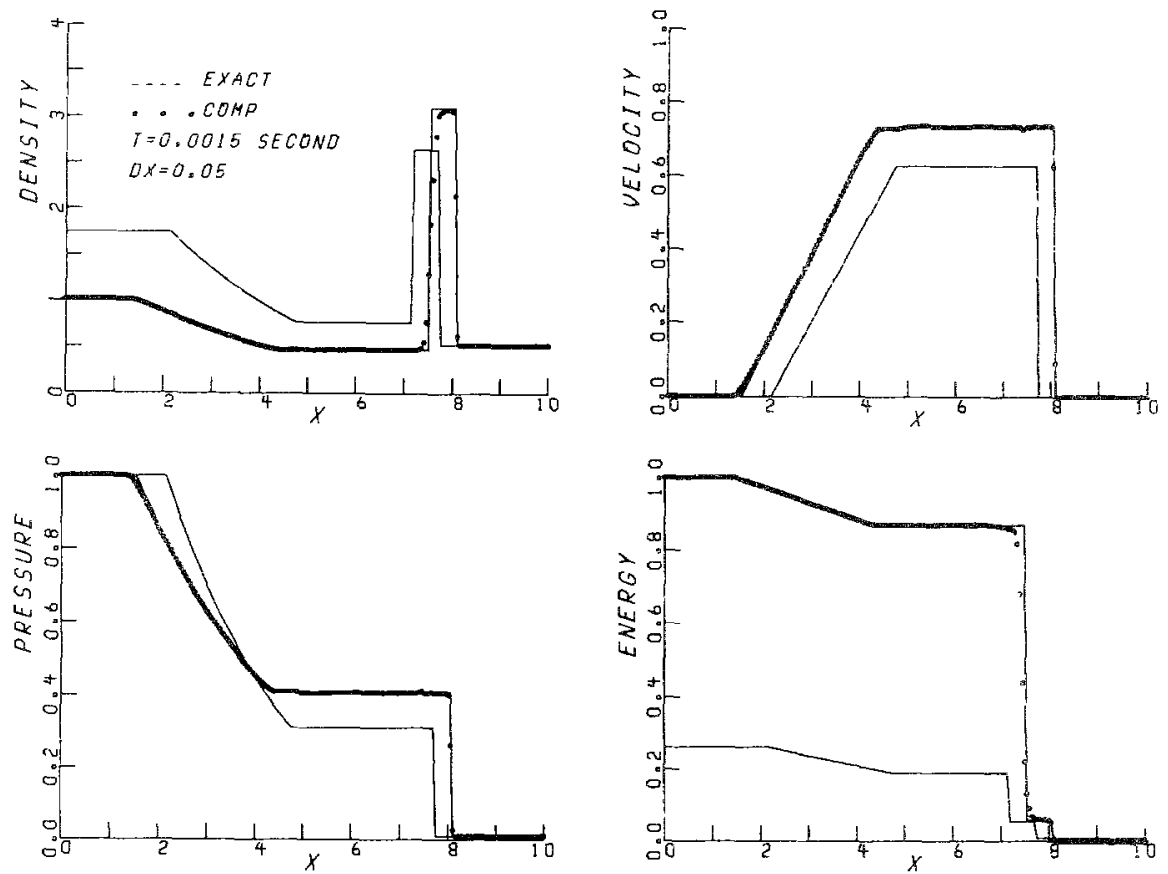

Fig. 3. Shock tube problem. Van Leer flux-vector splitting. 
gas, but accompanied by slight oscillations. Among all three splittings, the Roe scheme seems to give the best results, especially near the contact discontinuity.

The species molar fractions obtained by the Roe splitting are shown in Fig. 4. The sharp peak in the NO molar fraction is a numerical result produced by the smearing of internal energy at the contact discontinuity. This is because the NO molar fraction is a very strong, non-monotone function of temperature (internal energy) and is most stable at some intermediate temperature across the "numerical" contact discontinuity. It is seen that a large amount of recovery of $\mathrm{O}_{2}$ and $\mathrm{N}_{2}$ from $\mathrm{O}$ and $\mathrm{N}$ occurs across the contact due to the large temperature drop, while there is only a minute change across the shock. This is also the case for electrons, $\mathrm{N}^{+}$ and $\mathrm{NO}^{-}$.

The results of excluding the real-gas effect are also displayed for air with the same initial pressure and temperature as well as at the same final $t(=0.0015 \mathrm{~s})$. This amounts to taking $p=0.4 \rho e$ for the EOS. Significant dissociation and ionization take place at high temperatures in the real gas, thereby yielding a much larger specific internal energy and lower density than in the ideal gas. The shock and rarefaction wave in the ideal gas are travelling at a slower speed; the shock strength and the jump across the contact discontinuity also appear weaker.

Comparing the above results with those obtained by Grossman and Walters [8] with their real-gas Steger-Warming splitting is hindered by the lack of resolution
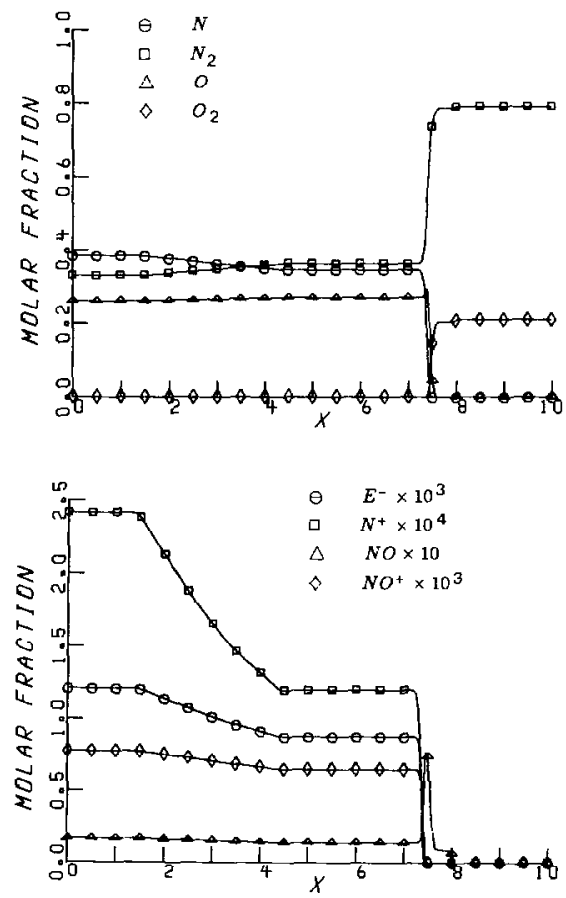

Fig. 4. Shock tube problem, molar fractions of species. 
in their figures. Specifically, only a few point values of the exact solution are indicated. Most obvious is that the post-shock density fails to reach the proper plateau value; this is caused by excessive smearing of the contact discontinuity following it and probably has led to an incorrect shock position. The smearing is a property of the TVD scheme and limiter used, rather than details in the flux-splitting formulas, so the results do not shed much light on the quality of the splitting.

Numerical results more similar to ours were obtained by Montagné, Yee, anc Vinokur [19], based on the split-flux formulas of [9]. The parameters of the shock-tube problems solved in [19] are different from those of Figs. $1-4$ and do not go beyond a temperature jump of a factor 7 at the contact surface; the results for the most severe case are not displayed. Disregarding this difference and some scheme-dependent errors, the results appear to be of comparable accuracy.

\subsection{Steady Nozzle Problem}

Calculations for steady real-gas flows in $1 \mathrm{D}$ convergent-divergent and divergent nozzles are given along with the exact ideal-gas solutions. The area distribution of the nozzle are listed below.
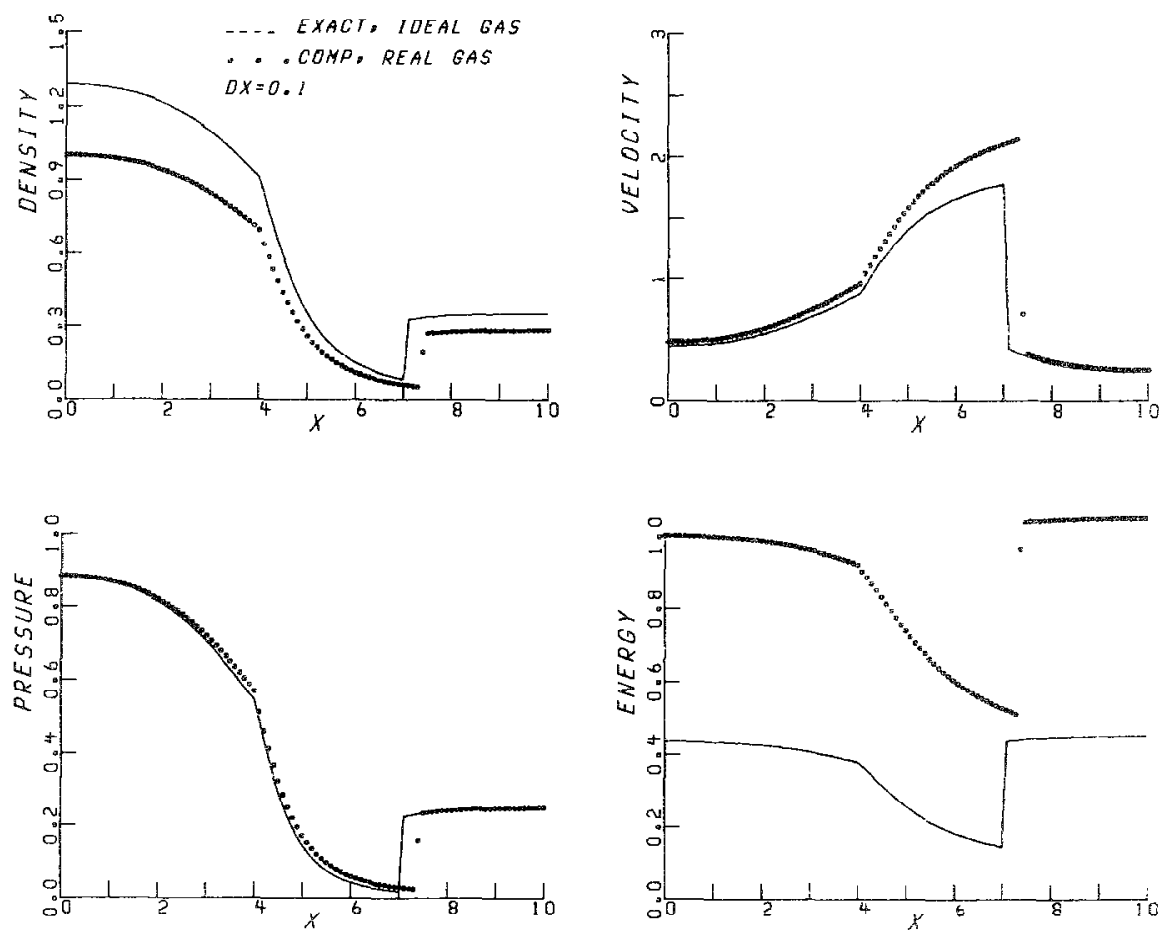

FIG. 5. Convergent-divergent nozzle problem, Roe flux-difference splitting. 

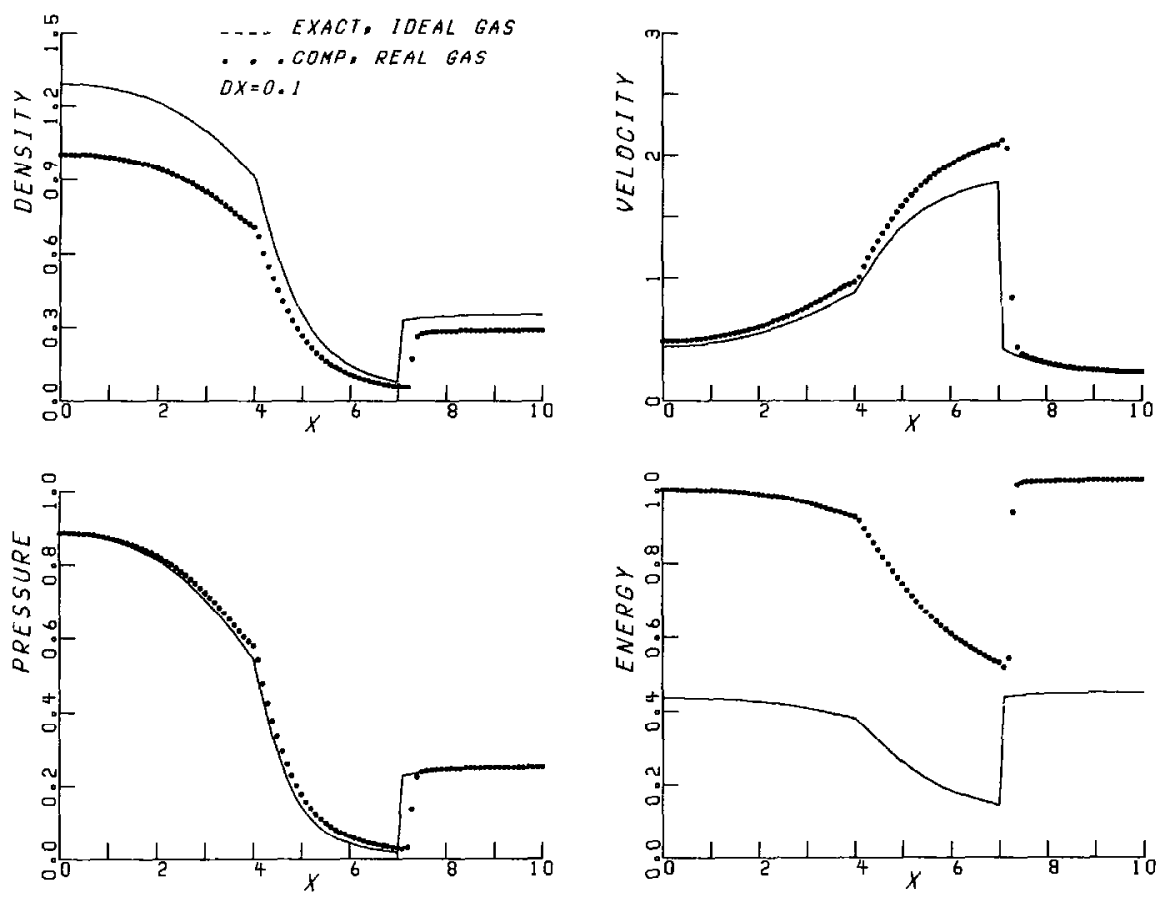

FIG. 6. Convergent divergent nozzle problem, Steger-Warming flux-vector splitting.
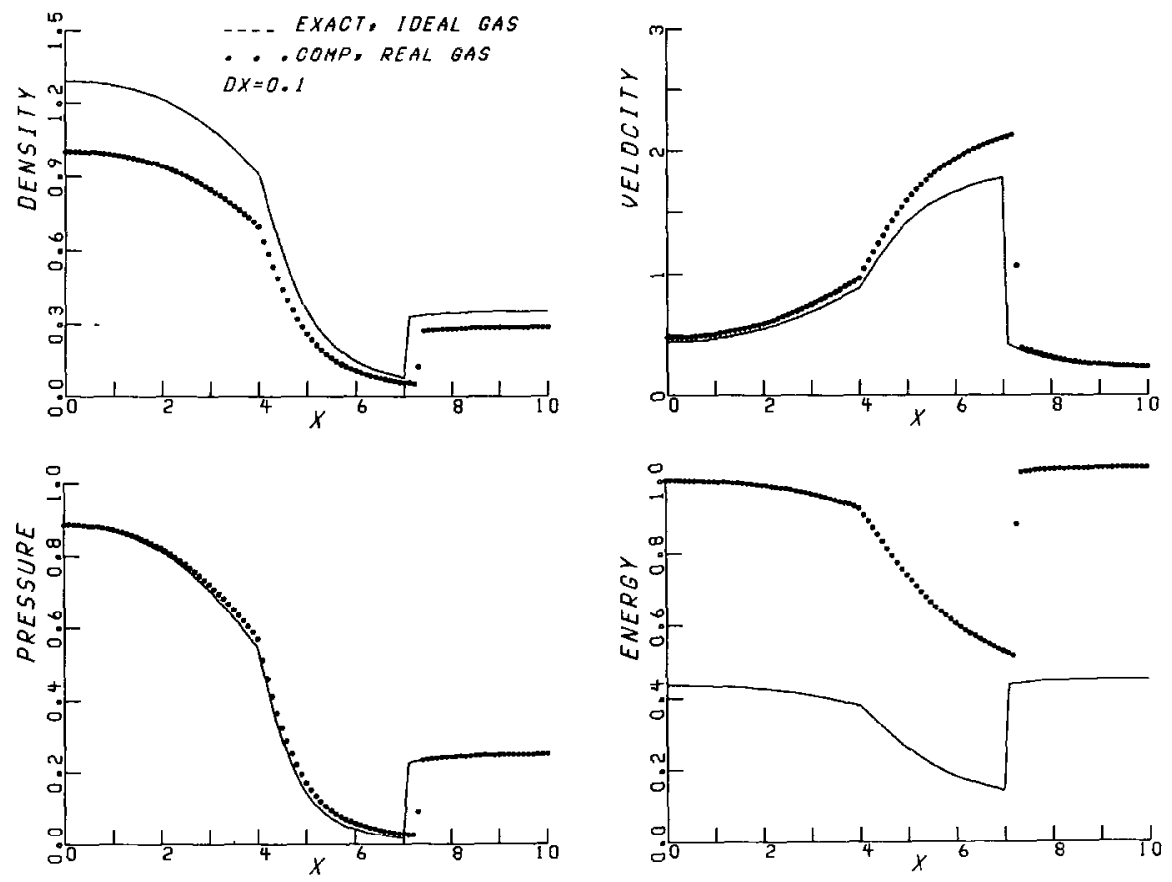

Fig. 7. Convergent-divergent nozzle problem, Van Leer flux-vector splitting. 

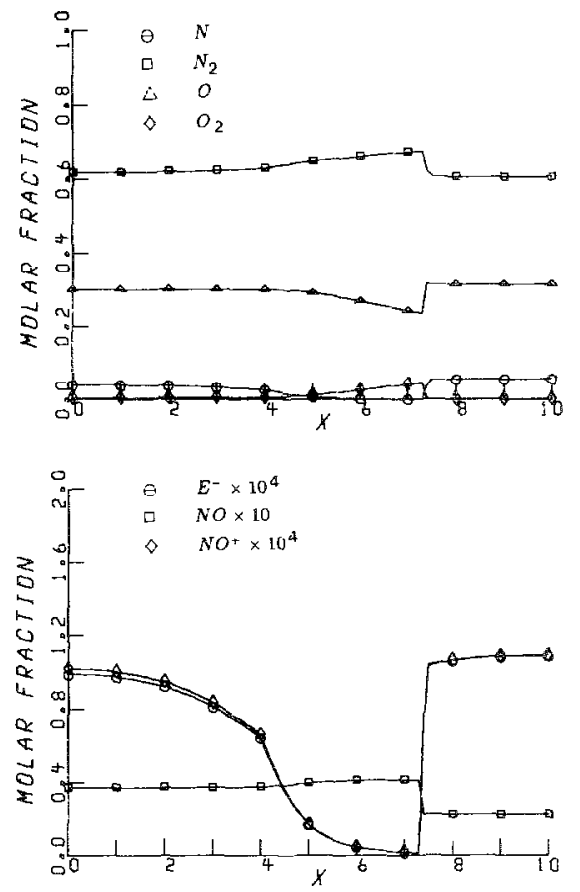

Fig. 8. Convergent-divergent nozzle problem, molar fractions of species. Roe splitting.
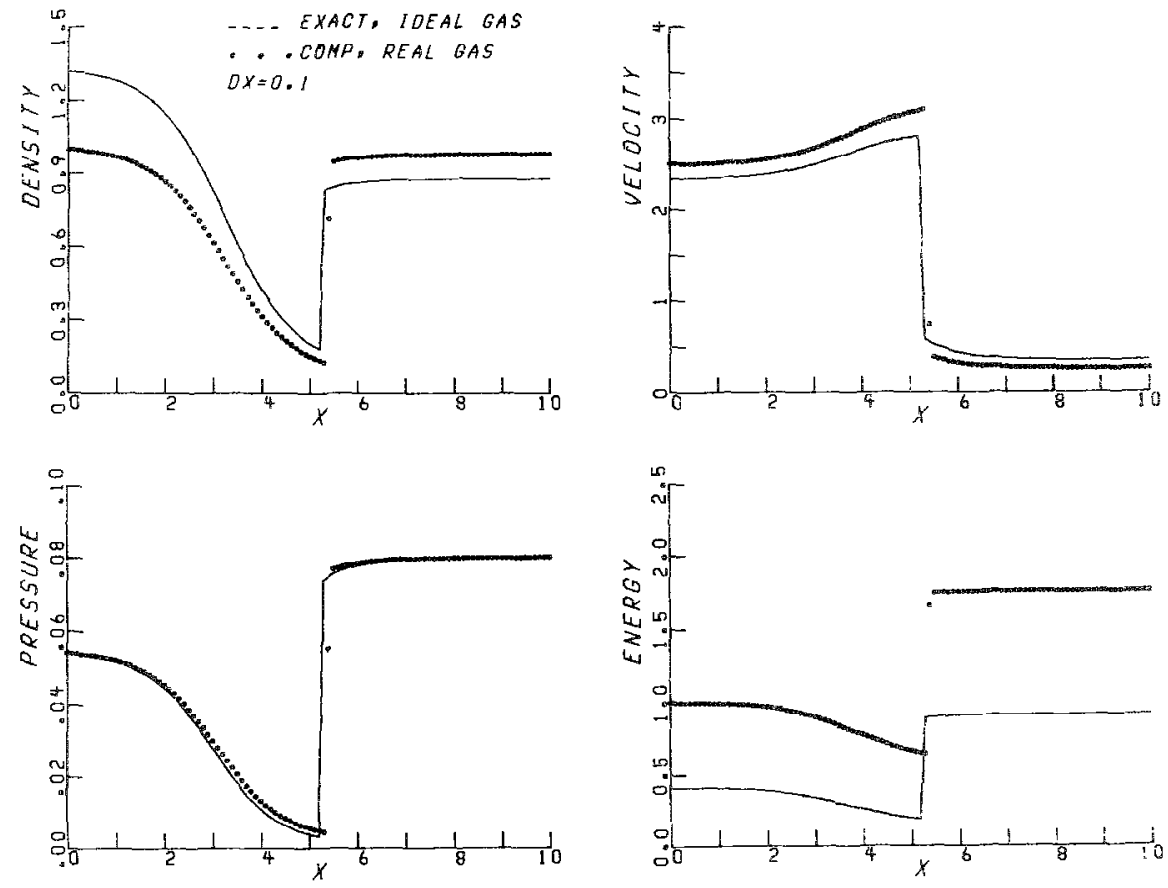

Fyg. 9. Divergent nozzle problem, Roe flux-difference splitting. 
Convergent-divergent nozzle:

$$
\begin{array}{ll}
A(x)=5.5-4.5 \cos \left(\frac{x-4}{6} \pi\right), & 4 \leqslant x \leqslant 10 \\
A(x)=1.2-0.2 \cos \left(\frac{x-4}{4} \pi\right), & x \leqslant 4 .
\end{array}
$$

Divergent nozzle:

$$
A(x)=5.5+4.5 \tanh (0.7 x-3.5), \quad 0 \leqslant x \leqslant 10 .
$$

Figures 5-7 show the results $p / p_{t \infty}, \rho / \rho_{x}, u / a_{x}$, and $e / e_{\infty}$ for the convergentdivergent nozzlc. Excellent agreement is achieved by all three schemes with monotone and sharp resolution across the shock, although Steger-Warming's is slightly more dissipative and smears over about two interior cells. The kink at the throat resulting from the discontinuity of area curvature, $A^{\prime \prime}(x)$, is resolved well. The ideal-gas shock wave in this case is slightly further upstream and the jumps in velocity and energy become weaker. As in the shock-tube problem, the high inflow temperature produces significant dissociation and ionization. Consequently, the internal energy more than doubles that of the ideal gas. The molar fraction of $\mathrm{N}$,
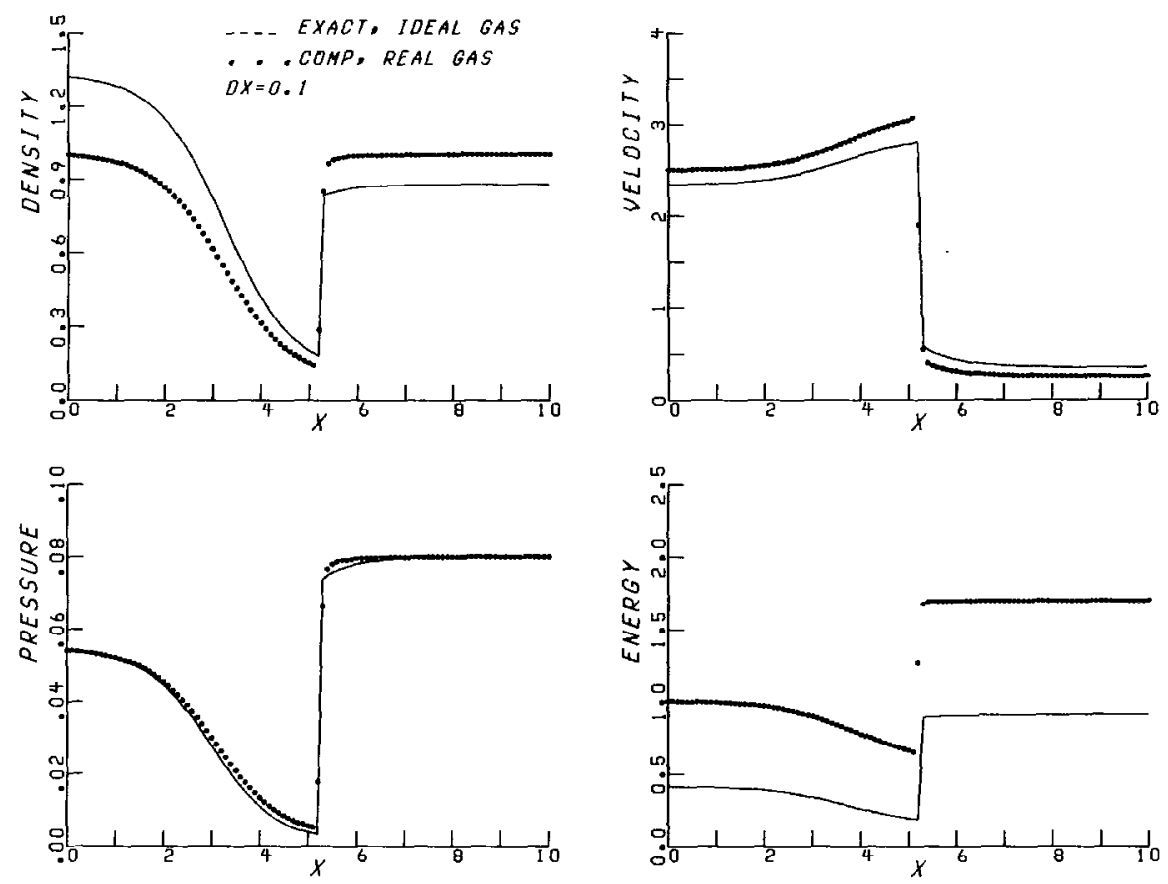

FIG. 10. Divergent nozzle problem, Steger-Warming flux-vector splitting. 

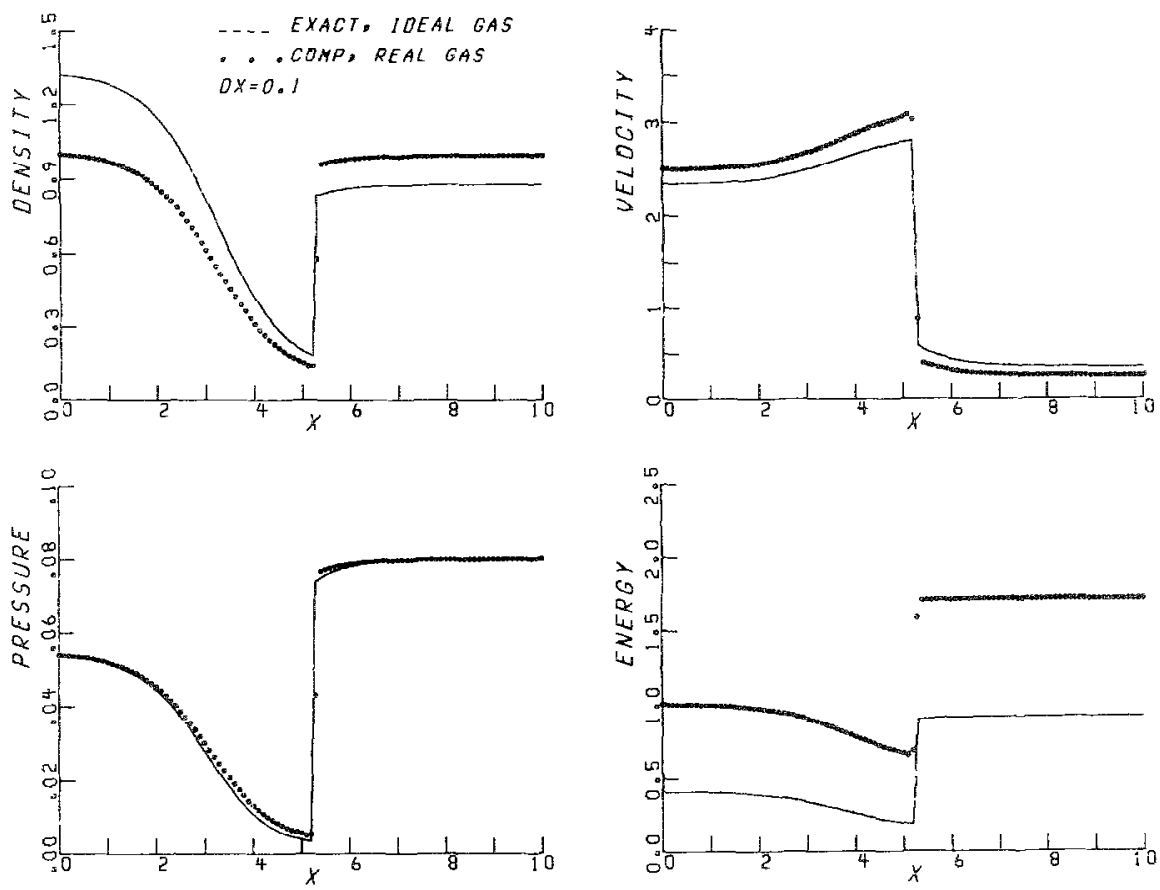

Fyg. 11. Divergent nozzle problem, Var Leer flux-rector splitting.
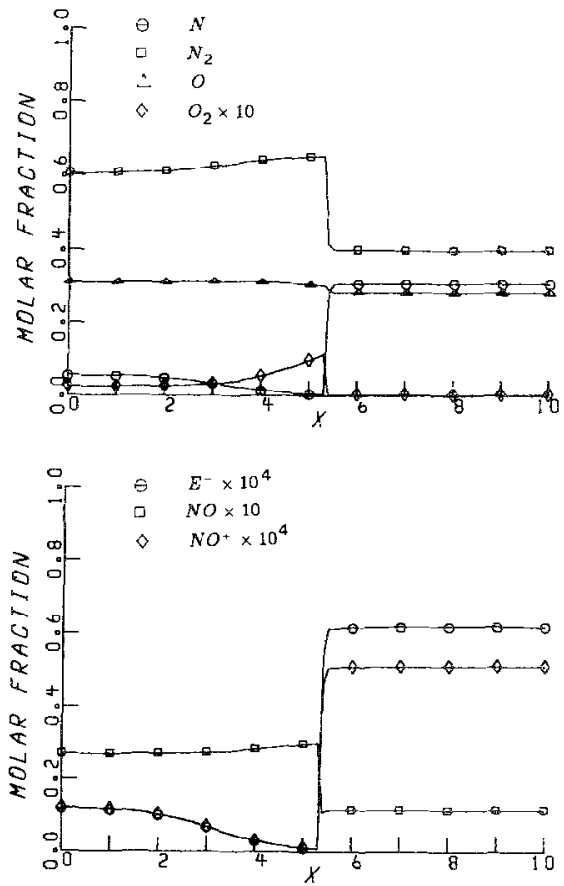

Fig. 12. Divergent nozzle problem, molar fractions of species. Roe spititing. 
O, electrons and $\mathrm{NO}^{+}$in Fig. 8 is decreased by the continued drop in temperature associated with the acceleration of the gas up until at the shock, but is increased abruptly by the shock. The molar fraction of $\mathrm{N}_{2}, \mathrm{O}_{2}$, and NO shows the reverse trend, due to the conservation of mass.

Calculations are also carried out for flows in the divergent nozzle; the results are given in Figs. 9-12. The shock wave is stronger than the above case, but the flow variations are generally similar. In this particular set of area geometry and flow parameters, the real-gas and ideal-gas shock locations are essentially identical.

\section{CONCLUDING REMARKS}

Care in deriving flux-vector and flux-difference splittings for a real gas pays off, as evidenced by the quality of the numerical results presented above. This is gratifying, because most formulas derived above, viz. the extensions of Van Leer's differentiablc flux-vector splitting and Roe's flux-difference splitting are close to being mathematically unique. The Steger-Warming splitting may be derived differently but we saw neither the way nor the need to do so.

From the numerical results for the shock-tube problem it is clear that the constraint on the width of a contact discontinuity is even more stringent for a real gas than for an ideal gas. The fictitious discrete states in a numerical profile representing a contact discontinuity trigger non-trivial chemistry, disturbing the pressure equilibrium that is so easily found for the ideal gas.

\section{REFERENCES}

1. S. K. Gudonov, Mat. Sb. 47, 271 (1959).

2. J. L. Steger AND R. F. Warming, J. Comput. Phys. 40, 263 (1981).

3. B. VAN LeER, Lecture Notes in Physics, Vol. 170 (Springer-Verlag, New York/Berlin, 1982), p. 507.

4. P. L. RoE, J. Comput. Phys. 43, 357 (1981).

5. S. OsHer, Math. Studies, Vol. 47 (North-Holland, Amsterdam. 1981), p. 179.

6. A. Harten, P. D. Lax. and B. van Leer, SIAM Rev. 25, 35 (1983).

7. P. Collela and H. M. Glaz, J. Comput. Phy's. 59, 264 (1985).

8. B. Grossman and R. W. Walters, AIAA paper 87-1117-CP, 1987 (unpublished).

9. M. VINOKUR AND Y. LiU, AIAA paper 88-0127, 1988 (unpublished).

10. P. Glaister, J. Comput. Phys. 74, 382 (1988).

11. M.-S. LIOU, AIAA paper 87-0355, 1987 (unpublished).

12. R. H. Sanders and K. H. Prendergast, Astrophys. J. 188, 489 (1974).

13. M.-S. LIOU AND B. VAN LeER, AIAA paper 88-0624, 1988 (unpublished).

14. B. van Leer, J. L. Thomas, P. L. Roe, and R. W. Newsome, AIAA paper 87-1104-CP, 1987 (unpublished).

15. D. Hänel, R. SchWANe, ANd G. Seider, AIAA paper 87-1105-CP, 1987 (unpublished).

16. W. K. Anderson, J. L. Thomas, ANd C. L. Rumsey, AIAA paper 87-1152-CP, 1987 (unpublished).

17. P. L. RoE, Ann. Rev. Fluid Mech. 18, 337 (1986).

18. S. GoRdon AND B. J. McBride, NASA SP-273, 1976 (unpublished).

19. J.-L. Montagní, H. C. Yee, And M. Vinokur, NASA TM 100004, 1987 (unpublished).

20. R. G. SMith, Trans. Amer. Math. Soc. 249, 1 (1979).

21. I. H. PARPIA, AIAA J. 26, 113 (1988). 